\author{
Contents lists available at www.cmro.in \\ JOURNAL OF CURRENT MEDICAL RESEARCH AND OPINION
}

Journal homepage: http://cmro.in/index.php/jcmro

\title{
"Public Health Hints: How To Best Breath And More Which Everyone Must Know And Use To Be Healthy!"--
}

\author{
Sameul A. Nigro ${ }^{1}$ \\ 1. Retired Assistant Clinical Professor of Psychiatry Case Western Reserve University.
}

\begin{abstract}
ARTICLE INFO
Corresponding Author:

Sameul A. Nigro

1. Retired Assistant Clinical Professor of Psychiatry Case Western Reserve University.

Keywords:oral hygiene, dental care, pulmonary hygiene, snoring, best breathing, choking, knee/back exercise, child drawing for mental health, obsessions, obesity, sore throat prevention, integration, prurience removal.
\end{abstract}

\begin{abstract}
Simple clinical health improving procedures are offered which should be promoted by public health organizations everywhere. These procedures are the result of discovering the cure for the author's severe laryngospasm disorder. Discovering the SAM prompted reflections on improving simple valuable self-care for all. All techniques are described and recommended for improving health care and comfortable living for everyone everywhere old enough to follow directions. They have to be daily practiced and made routine.
\end{abstract}

\section{INTRODUCTION}

Following these ten procedures will improve everyone's health.

\section{BEST MOUTH HYGIENE:}

Almost all contagious diseases from Strep Throat to viruses infect through the mouth--especially the gums (In fact, most likely all diseases have something to do with what we inhale or ingest). Basically, a clean mouth means strong gums which means better health. Teeth should be brushed twice a day--morning and bedtime with flossing before each time. Flossing firmly strengthens the gums. Teeth, gums and tongue need brushing, especially the gums which need massaging to stay firm and strong. The vibrating toothbrushes are best and give a 2 minute hygienic cleansing better than any manual brushing. The cost is readily covered by money saved on medications and less than the electronic game gadgets every one has. Mouth hygiene means not putting garbage into your mouth: Keep hands clean; avoid cigarettes, marihuana, inhalants, snorts, street drugs, or anything unclean. Keep every thing about and in your mouth clean and your gums strong. Mouth gargling and rinsing several times a day with a favorite over-the-counter mouthwash and/or occasionally with strong salt water solution (salt water kills most germs) is recommended. Finally, "best breathing" by the nose strengthens the mouth gums also by lessening gum dryness, and the nose is both a barrier and expeller of negative inhalants. Mouth hygiene is essential to good hospital care. (Also, as in 5 infra, topical dental antibiotic rub should be helpful in preventing infections if on steroids or if in a hospital or any where high in infectuous agents. In fact, a dab of triple antibiotic ointment in the nose and on teeth at the first sign of a cold might strengthen resistance. Also, a little dab of that vitamin filled skin cream helping your wrinkles might strengthen gums and nasal membranes.)

\section{BEST BREATHING:}

Make no mistake, there is a "best" way to breath (1). "Nasal inhalation" is the best way--It is more diaphragmatic requiring less muscle effort and thus more of the inhaled oxygen is available elsewhere in the body. However, "shutting your mouth" is obligatory for efficient and effective "nasal breathing"--laryngeal reflexes open better with "air into the nose" than with "air into the mouth." Mouth closure while breathing prevents dryness of mouth mucous membranes which work best when moist. A dry mouth because of mouth breathing does not protect well. Snoring dries the mouth, so practice sleeping by nasal inhaling and exhaling with shut mouth is essential. Choking basically is the erroneous intermingling of inhaling and swallowing which can be prevented and corrected by nasal breathing. Learn and practice the acronym "SAM": Shut mouth; Air in nose; Mouth cough (if choking or clearing throat) or just exhale (by mouth or 
preferably nose). The SAM must be practiced--it does not come naturally for most. Learn, practice, and teach the SAM--you will be "best breathing." Best Breathing is essential for good hospital care especially in intensive care units. Athletes gasping for air when out of the game should breath nasally for best re-oxygenation (I got this idea after watching professional hockey--and feeling quicker oxygen recovery when pushing my lawn mower as an 80 year old. It works!).

(By the way, the SAM works as a panic attack/temper/rage/confusion stopping technique by detachment from the source of emotionality to calmer self-awareness and improved oxygenation--"Panic" and all related are likely due to unconscious "not breathing" and one is low on oxygen. "Get more oxygen! Just do the SAM and think it through. Shut your mouth and breath through your nose!" Keep repeating this. See 7 below.)

\section{BEST COUGHING: "pulmonary hygiene!"}

The lungs never quit. They discretely expel unhealthy molecules inhaled so effectively that it is taken for granted and overlooked. Coughing is the body's way of expelling something definitely foreign and unwanted. Coughing is not just a symptom. It is nature's cleansing procedure. To make a cough more effective, do the SAM! First Shut the mouth, then inhale nasally as deeply as you can, and then really explode a mouth cough into your kerchief or elbow. That really should be done as personal health measure twice daily for 3 coughs after brushing your teeth each time. This cleans your lungs. If you feel a chest cold coming on, do it several more times whenever. It can help prevent the chest cold and can keep you healthier. At the first sign of a "cold", it should be done four times a day. It should be done after every cigarette by smokers. It does not take much reflection to conclude that lungs need help with "cleaning" the crude now being inhaled. This should help prevent lung cancer and most infectious diseases which enter the body through the lungs.

\section{BEST KNEE \& BACK EXERCISES:}

Walk $50+$ steps on your heels; or rock back and forth from one heel to the other. The paraspinal muscles (including the psoas) and hip ab\& ad -ductors are all tightened by walking on your heels. You will be surprised how the aches and pains often disappear after consistent heel walking (or rocking). The paraspinal muscles especially do not get exercised and it seems that the spinal cord system works better with stronger paraspinal muscles. I discovered this during my recovery from bilateral simultaneous knee replacements due to cartilage deterioration since "bends" during deep sea diving when in U.S. Navy Submarine Service. It worked and I found heel walking helped whenever I have had knee or back aches ever since. Walking on heels is almost a full body massage of back related muscles we hardly ever use in daily routines. Easiest to do while brushing your teeth. I am shocked at how this works if done at the first sign of back or knee discomfort.

5. SAVING TEETH: These are my experiences. At 78 years old, my lower left 2 nd molar begins to throb and hurt. The dentist checks with xrays finding nothing but recommends someone for extraction--"This is what is regularly done to relieve the pain and discomfort" (which I knew because of relatives' having many such extractions). However, the dentist did give me a trial of penicillin and higher fluoride toothpaste which did not work after two weeks. But I thought that the antibiotic by pills would never get concentrated about that tooth, so I bought topical triple antibiotic cream. I squeezed a little high fluoride toothpaste and the topical antibiotic on my finger and massaged them onto and around my hurting tooth. I did this after tooth brushing morning and night. My tooth cleared up and it has been 8 months with normal painless functioning. I still do the toothpaste/antibiotic massage every morning. It is merely one case, but it is so simple, inexpensive and it is still working! I have had successful outcomes with several others since including an old male who had three root canals but did not need a fourth after doing the antibiotic massage. Studies show that simple dental cavities are due to infection--occasional antibiotic massage may be preventive of most dental problems.

Also, dental antibiotic massage might help reduce infections if on steroids for any reason. I was briefly put on steroids for traumatic arthritis and could feel an increase in dental sensitivity, so I started my topical antibiotic dental massage until off the steroids. This makes one consider the "preventive" aspects of topical antibiotic dental massage for infections if in a hospital or wherever there are high concentrations of infectuous disease agents-or a dab into each nostril and on ones gums at the first sign of a cold might help defense against full cold syndrome.

If this works as well as I have experienced and witnessed, dentists will be shutting some of their schools.

6. SAVING CHILDRENS' MENTAL HEALTH: Doc Sam presented interactional drawings with children as an effective therapy in child psychiatry and mental health promoting technique for parents and their children. This was a Grand Rounds Presentation, Department of Psychiatry, Case Western Reserve University School of Medicine: "Therapeutic Sequential Drawings with Children -- a New Technique" 1970. He extended D. W. Winnicott's "squiggles" by taking turns with the child in the creation of the drawings. This helped and led the child to express the good, the bad, the ugly, the helpful for "that week" or month or any time interval. Any past or anticipated event could be focused on by interactional mutual drawing with the child creating, and reliving, an emotive event. While drawing, the therapeutic emoting and processing were far beyond simple "talking it out." The words used while drawing have more intense, deeper and material substance to the catharsis. Reproductions of series of drawings were provided with the stories briefly detailed. The presentation was then submitted as an article for the Journal of Pediatric and Adolescent Psychiatry. A jealous and anti-Catholic supervisor prevented its publication. She graduated the same year as Doc Sam from medical school, but got to complete her psychiatric training while Doc Sam served four years in the U.S. Navy. That qualified her to supervise him during his psychiatric residency. The director of the Department of Child Psychiatry made her tell Doc Sam of her preventing his article's publication. Other reviewers of the Journal of 
Pediatric and Adolescent Psychiatry contacted him about his "Squiggles" article asking his support for their individual plans to promulgate such. Doc Sam believes that parents and others can do the Squiggle drawing effort with their children on a regular basis with great emotional benefit and positive interactional relationship enhancement. "Let's draw the best thing that happened this week." "Let's draw the worst thing that happened." "Let us draw_____ ("your emergency room visit!" for example). Doc Sam believes this is not only a therapeutic technique for physicians wanting to help a child work through emotions, but a universal mental health parenting technique helping positive child development and self understanding in the family setting. Universally, parents should do this with each child each week. Doc Sam believes this will improve all child development and family togetherness.

\section{REDUCING OBESITY AND OTHER OBSESSIONS:}

"distract from obsessions"

By doing the SAM gently and repeatedly, when the urge hits to eat or do one's obesssion, and repeating the Catholic Mantra of "life, sacrifice, virtue, love, humanity, peace, freedom, and death without fear" over and over, you may be able to gradually free yourself from your habituation/obsession. It takes time and practice but most can be helped by the extra oxygen of the SAM and the distraction of naming the ultimate human purposes. (See 2 above.)

8. STOPPING SNORING: Snoring means you are mouth breathing. Like Pavlov's dog, condition technique should work. Jostle the snorer shaking his shoulder. At the same time say: "Shut your mouth (you can gently push his chin with other hand) and breath through your nose only and roll over". Repeat until he does. In time, with repetition, he will likely be conditioned to do it all as soon as you put your hand on his shoulder. Snoring is from the mouth. With the mouth closed, there is no snoring. Breathing through the nose gets more oxygen and is healthier than mouth breathing.

9. STOP THAT SORE THROAT: At the first "soreness" of the throat...that early sharp pain with normal swallowing...beginsalt water gargles! Take small cup of water and add tablespoon + of salt. Gulp into your throat without swallowing. Swirl around with head held back and let flow to back of throat and then gargle as best you can. Swirl around again and spit out into the sink. Do over and over until cup is empty. Repeat every 4-6 hours until sore throat fades. See your doctor if persists over 24 hours. Hopefully the salt will kill the reasons for the sore throat. And do the pulmonary hygiene ( 3 above).

10. PANHUMANITY: Live "full" humanity as best you can. "PAN" is redundant, but needed because color, ethnicity, race, religion, groupthink tend to make all forget that "humanity" is all of us!As my Italianate father would say banging the table: "We are all the same! We are all the same! Even when we proclaim our differences!" You cannot fight racism with your own racism. You cannot fight color with color. You cannot fight sub-culture with sub-culture. To embrace less than "content of character" is to be part of the problem rather than the solution. Color, race, sex, geographic heritage et cetera are as important as height and weight. As Martin Luther King, Jr. encouraged: "Content of character" is how to present yourself virtuously and judge virtues of others. We must identify as HUMANS: African, Asian, Caucasian, Hispanic, Negro, and by other words which bring the personhood of transcendent human history: Life, Sacrifice, Virtue, Love, Humanity, Peace, Freedom, and Natural Death without Fear by commitment to Life, Liberty and the Pursuit of Happiness--All these being basic ultimate human purposes. Anything else will almost always fail because it is not fully human because it ignores the content of one's character. Content of character, asDr. King stated, MEANS: at least, the virtues of prudence, justice, temperance and fortitude--which are the only ways to act and how to judge others. If you really want to be healthy and help everybody's health, stop promoting that which identifies less than fully human. Color et cetera hardens the heart and dissolves the brain.

11. REMOVING PRURIENCE (3): As Freud proved, sex makes you crazy. Thus, everyone needs the words to reduce seductive genital mania in a culture which uses sex to create excitement, interest and imitation even though it is pollution against nature. In Nature, sex is consistent with normal functioning and reproduction. It is not "anything/ anywhere" as humans now abuse themselves. Until now, human cultures have always had rules to keep sex for opposite sexed adults at a time consistent with reproduction. "Marriage" was the psychosocial pheromone for humans, just as biochemical pheromones kept sex "reproductive" for subhumans (got a cat?). Be glad humans do not have biochemical pheromones because there would be no "love" between us. Here are the words to remove prurience for everyone's better health:

(1) Seeing shapely buttocks, call them "anus protectors" or "bowel movement protectors".

(2) Seeing breast presented seductively, call them"milk, cancer, sweat, fat, pontoon, hanging, useless chest glands."

(3) Seeing the vagina, call it a "baby blood hole which has a urine hole in front of it, a feces hole behind it and is filled with blood $1 / 4$ of the time."

(4) Seeing the penis, call it the "ud(urine director) because 99 percent of the time, the ud (nee' penis) is used to urinate in the direction wanted by the male and should always be called the 'urine director' ('ud') instead of 'penis' (the latter word now applicable only to normal sexual intercourse).

(5) Seeing cleavage, call it "female mooning."

(6) Seeing pubic hair, call it "friction absorbers so the skin is not injured during intercourse."

(7) Seeing orgasm outside of normal intercourse, call it "masturbation or sexcretion which is squirting for males and sliming for females outside of normal sexual functioning consistent with reproduction, and thus is unnatural and therefore "pollution". Remember that sexual relief is basically selfishness or a form of natural excretion (like "wet-dreams") which should not become enslavingways of genital manias or obsessive-compulsive disorders. Sexual squirting for men and sexual sliming for women are body excretory acts accompanying nature's way of starting a baby-neither squirting nor sliming should be so important that one loses one's identity and life because of them. 
(8) Remember, my colleagues words as he went back to his country to find a woman to marry: "American women are really something. You can always get a handjob, a blowjob, or a quick screw--It is as significant for our relationship as my taking a leak. They are harem-feminists." Do not create haremfeminist members. Do not be part of the local feminist harem.

(9) Remember that "Non-marital sexual activity is nothing but helping each other'go to the bathroom' ('toileting' or 'sexleting' or 'sexcreting') and that 'pornography' is nothing but public toileting and public excretion best seen as 'a bunch of dogs licking each others' rear ends'."

(10) Help us remember not only that "we are born between feces and urine" but that we procreate between feces and urine too-And please, God, help us remember all this so we do not become crazy about sex, any more than crazy about urinating, defecating or menstruating. Remind us that for all animals on the planet, sex is a simple biological function to create life-it is not recreation, entertainment or a game. Help us remember that the fantasy filled press and media (liar-papers and unrealvision) culture of celebrated indecency and dishonor is actually the creation and promotion of non-being (which is also a good definition of "evil"). Remind us that for mankind, sexual activity is for unition and reproduction in a spiritual and marital relationship for family life, and that all other sexuality is sliming and squirting excretory pollution when outside of marriage. Help all know that almost all illnesses enter the body through the mouth and keeping ones mouth clean and healthy makes for a long life (and thus the mouth should not be used for toileting!). Help young people know that they will fall in and out of love with "broken heart" feelings many times before finding the right person to marry, so pre-marital sex is not smart but is to sell one's self out to another when the smartest approach is to never let the other party think you care very much until there is a mutual marriage commitment-and even then to help each other "go to the bathroom" is almost always a violation of the future marriage vows and a betrayal of one's future spouse. Help young people to know that, even though it is the best feeling in the world, "puppy luv" makes you stupid and a victim. Regardless, thank God for the sexual creativeness of our lives in marriage and Nature. And especially, thank God for making sex pleasurable, otherwise there would be no human race and we would likely not touch each other with ten foot poles. Help us remember that sex is sacred in marriage and that extramarital sex is excretion or satire. Help us remember that lives are saved by Natural sex and lost by non-marital sex. And by practicing these ten phrases, you will be less likely to be made crazy by sex and can help others also. You will not be manipulated by the dirty old men, the gay genital maniacs, and the haremfeminists in charge of the press \& media.

(Check "authors" for many of my other articles on MEDCRAVE.)

\section{References:}

(1) Nigro SA (2015) The "Best" Way to Breath. J Lung PulmRespir Res 2(1): $00033 . \quad$ DOI:
(2) Related references are available at Medcrave under my name.

(3) Nigro, SA, Soul of the Earth.

10.15406/jlprr.2015.02.00033 